\title{
Association between adverse pregnancy outcome and imbalance in angiogenic regulators and oxidative stress biomarkers in gestational hypertension and preeclampsia
}

\author{
Cornelius A. Turpin², Samuel A. Sakyi ${ }^{1,3^{*}}$, William KBA Owiredu' ${ }^{1}$, Richard KD. Ephraim ${ }^{4}$ and Enoch O. Anto ${ }^{1}$
}

\begin{abstract}
Background: Gestational hypertension (GH) and Preeclampsia, (PE) are the most complicated amongst hypertensive disorders of pregnancy. The mechanism that links hypertension in pregnancy to adverse maternal outcomes is not fully understood though some relate this to endothelial dysfunction originating from an imbalanced angiogenic regulators and oxidative stress biomarkers. This study assessed the correlation between angiogenic regulators and oxidative stress biomarker levels with adverse pregnancy outcomes among GH and PE participants.

Methods: A cohort of pregnant women who received antenatal care at the Obstetrics and Gynaecology department of the Komfo Anokye Teaching Hospital (KATH) were followed. During their antenatal visits, 100 developed PE and 70 developed GE, of these, 50 PE and $50 \mathrm{GH}$ gave informed consent. Their blood samples were taken at time of diagnosis and $48 \mathrm{~h}$ post-partum. 50 other aged-matched women who did not develop neither GH nor PE were selected as controls. Placental growth factor (PLGF), soluble fms-like tyrosine kinase 1 (sFlt-1) and 8-epi-prostaglandin F2alpha (8-epi-PGF2a) levels were estimated by ELISA and total antioxidant capacity (T-AOC) was measured spectrophotometrically. Graphpad Prism was used for data analysis.
\end{abstract}

Results: Median levels of sFlt-1, 8-epi-PGF2a and sFlt-1/PLGF were elevated among participants with PE co-existing with intrauterine fetal death (IUFD), placental abruptio, placental previa, HELLP syndrome and intrauterine growth restriction (IUGR) compared to PE without adverse outcomes ( $p=0.041, p=0.005, p=0.0002)$. Levels of PLGF, T-AOC and PLGF/ sFlt-1 were significantly reduced among participants with PE co-existing with IUFD, placental abruptio, placental previa, HELLP syndrome and IUGR compared to PE without adverse outcomes ( $p=0.0013, p=0.006, p<0.0001)$. A significant negative correlation of IUGR $(p=0.0030 ; p<0.0001)$, placental abruptio $(p<0.0001 ; p<0.0001)$, IUFD $(p<0.0001 ; p<0.0001)$, stillbirth ( $p=0.0183$ and $p<0.000)$, and postpartum haemorrhage (PPH) $(p=0.0420 ; p=0.0044)$ were associated with both PLGF and T-AOC whilst a significant positive correlation of IUGR, placental abruptio $(p<0.0001$; $p<0.0001)$, IUFD $(p<0.0001 ; p<0.0001)$, stillbirth $(p<0.0001 ; p<0.0001)$, and PPH $(p=0.0043 ; p=0.0039)$ were observed with both sFlt-1 and 8-epi-PGF2a in PE.

Conclusions: Imbalance in the levels of angiogenic regulators and oxidative stress biomarkers correlates with adverse pregnancy outcomes among PE participants. Early identification of these imbalance would alert health care givers in anticipation of adverse pregnancy outcome and thus increased surveillance during pregnancy and parturition and measures to ameliorate the adverse outcome.

\footnotetext{
* Correspondence: sasakyi.chs@knust.edu.gh

'Department of Molecular Medicine, School of Medical Sciences, Kwame

Nkrumah University of Science and Technology (KNUST), Kumasi, Ghana

${ }^{3}$ Department of Bacteriology, Noguchi Memorial Institute for Medical

Research, Accra, Ghana

Full list of author information is available at the end of the article
} 


\section{Background}

Preeclampsia, the most complicated amongst hypertensive disorders of pregnancy is enigmatic due to its multisystemic nature and associated adverse pregnancy outcomes [1]. The mechanism of how some pregnancies lead to adverse maternal outcome is not fully understood though endothelial dysfunction, advanced maternal age and gestational hypertension has been suggested to play a role [2, 3]. Adverse pregnancy outcome is any event which reduces the chance of having a healthy baby and includes: stillbirth, preterm delivery, foetal anomalies, intrauterine growth retardation (IUGR), intrauterine fetal death (IUFD) and small for gestational age (SGA) infants. These adverse outcomes have been linked with preeclampsia [4]. Despite this link, little or not much research have explored the association between both antepartum and postpartum adverse pregnancy outcomes, and angiogenic regulators and oxidative stress biomarker levels. Placental released proteins such as soluble fms-like tyrosine kinase-1 (sFlt1) and placental growth factor (PLGF) are angiogenic regulators which levels varies considerably in circulation during pregnancy [5]. A previous published study found an increased levels of sFlt-1 and a decreased PLGF concentrations to be associated with IUGR, spontaneous preterm birth and stillbirth [6] whilst other study found an inconsistent result [7]. Pregnancies complicated by low birth weight (LBW), spontaneous preterm delivery, and small for gestational age (SGA) infants have been shown to have reduced antioxidant and increased pro-oxidant levels, although no correlational data have been explored to establish this link [8]. Ghana's attempt to achieve the millennium development goals (MDG) four and five has not been successful due to high incidence of adverse pregnancy outcomes. The association between angiogenic regulators and oxidative stress levels may share a common etiological pathway, moreover, correlational data between angiogenic regulators, oxidative stress biomarker levels and adverse pregnancy outcome in PE have not been published in any previous studies. It is against this background that this study seek to assess the correlation between angiogenic regulators and oxidative stress biomarker levels with adverse outcomes in gestational and preeclamptic women.

\section{Methods}

\section{Study design and study setting}

This hospital based prospective cohort study was carried out from April to November, 2014 at the Obstetrics and Gynaecology (O \& G) department of KATH in Kumasi. Kumasi is in the Ashanti Region of Ghana and has an average population of 4,780,380 (Ghana Statistical service, 2012). KATH is the second largest tertiary hospital in Ghana with a thousand (1000) bed capacity. It serves as a major referral centre for the middle belt and northern part of Ghana. The hospital also receives referrals from other regions and this gives fair representation of the Ghanaians population.

\section{Selection of participants}

A cohort of pregnant women who patronize antenatal services at the O\&G department of KATH were followed. During their periodic visits, 100 developed PE, and 70 developed GH. Some were lost to follow ups and some refused to give an informed consent. Finally, 50 $\mathrm{GH}$, and 50 PE pregnant women gave written informed consent and their blood samples were taken at the time of diagnosis and $48 \mathrm{~h}$ postpartum. 50 other agedmatched pregnant women who did not develop neither GH nor PE were used as normal controls. The diagnosis of hypertensive disorders of pregnancy was done by qualified Obstetrician/Gynaecologist using the National High Blood Pressure Education Program Working Group diagnostic criteria (NBPEPWG, 2000). Information relating to obstetric and demographic characteristics were obtained from record reviews of hospital database and structured closed ended questionnaires. Preeclampsia was defined as the onset, after 20 weeks of gestation for both hypertension $(>140 / 90 \mathrm{mmHg})$ and dipstick proteinuria $(>+0.3 \mathrm{~g} / \mathrm{l})$. Gestational hypertension was defined as hypertension occurring after 20 weeks of gestation, without dipstick proteinuria [9].

\section{Inclusion criteria and exclusion criteria}

Nulliparous and multiparous pregnant women aged $18-40$ years, within the gestational age of $\geq 20-40$ weeks with singleton pregnancy were included in this study. Participants previously diagnosed with chronic hypertension, heart disease, diabetes mellitus, renal disease or are on antihypertensive therapy as well as those who were unable to give informed consent were excluded from the study.

\section{BP measurements}

Trained personnel used a mercury sphygmomanometer (Accoson, England) and a stethoscope $\left(3 \mathrm{M}^{\mathrm{m}}\right.$ Littmann $^{\circ}$ Stethoscopes, USA) to measure the blood pressure of participants in accordance with recommendations of the American Heart Association [10]. The procedure was repeated for each patient between 5 and10 min. Mean values of duplicate measurements were recorded as the blood pressure to the nearest $2.0 \mathrm{mmHg}$.

\section{Urine sample collection and estimation of proteinuria}

Participants provided $10-20 \mathrm{ml}$ freshly voided early morning urine in clean, wide mouth and leak proof containers. Semi quantitative proteinuria was immediately assessed using dipstick (URIT $2 \mathrm{~V}^{\mathrm{PG}}$ Medical electronic Co., Ltd. China). Proteinuria was defined as the presence of urinary protein in concentrations more than $0.3 \mathrm{~g} / \mathrm{l}$ 
or $\geq+$ using the semi-quantitative colour scale on the urine reagent dipstick on urine dipstick [9].

\section{Ethical consideration}

Ethical approval was granted by the Committee on Human Research, Publications and Ethics (CHRPE) (CHRPE/AP/ 365/14), School of Medical Sciences, Kwame Nkrumah University of Science \& Technology (KNUST) and the Research and Development Unit of the Komfo Anokye Teaching Hospital (KATH). Patients who gave informed written consent were involved in the study. All data were de-identified before analysis.

\section{Blood sample collection}

$10 \mathrm{mls}$ of venous blood sample was collected from each participants. Blood was dispensed into serum separator tubes and centrifuged (Nüve NF 200, Germany) at $7000 \mathrm{rpm}$ for $15 \mathrm{~min}$. Serum was aliquoted under sterile conditions and stored at $-80{ }^{\circ} \mathrm{C}$ (Thermo Scientific ${ }^{\text {max }}$ Revco $^{\text {TM }}$ UxF - Ultra-Low Temperature Freezers, USA) until assay.

\section{Measurement of sFlt-1, PLGF and 8-epi-PGF2a}

Serum levels of sFlt-1, PLGF and 8 -epi-PGF2 $\alpha$ were measured in duplicate using commercially available ELISA kits from R\&D System Inc. (Minneapolis, MN USA). The optical density was measured at $450 \mathrm{~nm}$ using microplate ELISA reader (Mindray MR-96A). The plasma levels of each factor were calculated using standard curves derived from a known concentration of the respective recombinant factors.

\section{Total antioxidant capacity (T-AOC) assay}

Total antioxidant capacity (TAOC) reagents was obtained from Green stone Swiss Co., Ltd, China and serum levels were estimated spectrophotometrically (Mindray BA$88 \mathrm{~A}$, China) at $593 \mathrm{~nm}$. This assay was measured based on the ferric reducing ability of plasma (FRAP) method. The measurement of the ferric reducing ability of plasma (FRAP) was done by the assay protocol as described by Benzie and Strain, (1999). All samples were analyzed in triplicate.

\section{Definition of obstetric terms}

IUGR was defined as poor growth of a fetus while in the mother's womb during pregnancy [11]. Severe preeclampsia was defined as a systolic blood pressure (SBP) $\geq 160 \mathrm{mmHg}$ or a diastolic blood pressure (DBP) $\geq 110 \mathrm{mmHg}$ on two occasions recorded 6-h apart in association with proteinuria $(\geq+3)$ [12]. Preterm delivery was defined as delivery before 37 completed weeks of gestation [13]. Stillbirth was defined based on International Classification of Diseases, 10th revision (ICD-10) as "death prior to the complete expulsion or extraction from its mother of a product of conception, with respective to the duration of pregnancy [14]. IUFD was defined as the fetal death (where there is no sign of life) at equal or more than 20 weeks of gestation and/or birth weight of equal or more than 500 gram [15]. An abruptio placenta was defined as premature separation of normally placed placenta from uterine wall after 20 weeks of gestation and prior to birth [16]. Placenta previa was defined as implantation of the placenta over or near the internal orifices of the cervix after 20 weeks gestation prior to transvaginal or abdominal ultrasonography [17]. Premature pre-rupture of membranes (PPROM) was defined as rupture of the membrane of the amniotic sac and chorion occurring before the onset of labour [18].

\section{Statistical analysis}

Statistical analysis was performed using software Graphpad Prism, version 5.0 (Graph Pad Software Inc., Los Angeles) for windows. Comparison between contingency variables was performed using Chi-square test. Data were reported as mean \pm standard deviation (SD) for continuous data, as median (interquartile range) for non-parametric parameters (biomarkers) and as a frequency (percentage) for categorical data. The correlations between angiogenic factors and oxidative stress and adverse outcome were determined by Spearman correlation analysis. Statistical significance was accepted at $p<0.05$ for all comparisons.

\section{Results}

\section{Socio-demographic, obstetric and clinical characteristic}

The mean age of the general study participant was 29.78 years. Greater proportion $(83.3 \%)$ of the pregnant women were married whilst $16.7 \%$ were singles. The percentage of married participants with PE $(80.0 \%)$ and GH (76.0 \%) were significantly lower compared to the normal pregnant women $(94.0 \%)(p=0.0173)$. Among the $55.3 \%$ of the participant who had completed primary education, $72.0 \%$ developed PE and $58.0 \%$ had GH compared to $36.0 \%$ among normal pregnant women $(p=0.0052)$. A higher percentage $(42.7 \%)$ of the participants were nulliparous whilst 35.3 and $22.0 \%$ were multiparous and primiparous respectively. Majority of the participants were multigravida 59/150 (39.3 \%) of which $52.0 \%$ developed GH and $24.0 \%$ had PE. Significantly higher proportion of preeclamptic participants than GH and NP had spontaneous abortion (64.0\% vs $44.0 \%$ vs $42.0 \% ; p=0.0288)$, family history of hypertension (34.0\% vs $8.0 \%$ vs 2.0 ; $p<0.0001)$ and previous caesarean section (48.0\% vs $18.0 \%$ vs $12.0 \% ; p<0.0001)$. Participants with PE and $\mathrm{GH}$ had a significantly higher mean levels of systolic blood pressure (SBP) $(p=0.0020)$, diastolic blood pressure (DBP) $(p=0.0079)$ and proteinuria $(p<0.0001)$ compared to normotensive pregnant women $(p<0.05)$ (Table 1). 
Table 1 Sociodemographic, obstetric and clinical characteristics of study Participants

\begin{tabular}{|c|c|c|c|c|c|}
\hline Variables & $\begin{array}{l}\text { Total } \\
(n=150)\end{array}$ & $\begin{array}{l}\mathrm{NP} \\
(n=50)\end{array}$ & $\begin{array}{l}\mathrm{GH} \\
(n=50)\end{array}$ & $\begin{array}{l}\mathrm{PE} \\
(n=50)\end{array}$ & $p$-value \\
\hline Mean age (years) & $29.8 \pm 0.4$ & $30.79 \pm 0.7$ & $30.49 \pm 0.8$ & $28.85 \pm 0.6$ & 0.8992 \\
\hline Marital status & & & & & 0.0179 \\
\hline Single & 25 (16.7\%) & $3(6.0 \%)$ & 12 (24.0\%) & 10 (20.0\%) & \\
\hline Married & $125(83.3 \%)$ & 47 (94.0\%) & 38 (76.0 \%) & 40 (80.0\%) & \\
\hline Level of education & & & & & 0.0052 \\
\hline No education & $5(3.3 \%)$ & $0(0.0 \%)$ & $2(4.0 \%)$ & $3(6.0 \%)$ & \\
\hline Primary & 83 (55.3\%) & 18 (36.0 \%) & 29 (58.0 \%) & 36 (72.0 \%) & \\
\hline Secondary & 37 (24.7\%) & $12(24.0 \%)$ & 19 (38.0\%) & $6(12.0 \%)$ & \\
\hline Tertiary & 28 (18.6 \%) & 20 (40.0\%) & $3(6.0 \%)$ & $5(10.0 \%)$ & \\
\hline GA at baseline & $24.1 \pm 4.0$ & $24.3 \pm 5.1$ & $23.81 \pm 4.4$ & $24.39 \pm 2.6$ & 0.9102 \\
\hline GA at delivery & $37.0 \pm 0.7$ & $38.55 \pm 0.4$ & $36.91 \pm 0.4$ & $35.62 \pm 0.3$ & 0.5711 \\
\hline Parity & & & & & 0.7601 \\
\hline nulliparous & 64 (42.7 \%) & 20 (40.0\%) & 19 (38.0\%) & $25(50.0 \%)$ & \\
\hline primiparous & 33 (22.0 \%) & $12(24.0 \%)$ & 11 (22.0 \%) & 10 (20.0\%) & \\
\hline multiparous & $53(35.3 \%)$ & 18 (36.0 \%) & 20 (40.0\%) & 15 (30.0 \%) & \\
\hline Gravidity & & & & & 0.0501 \\
\hline primigravida & 44 (29.3\%) & $12(24.0 \%)$ & 14 (28.0\%) & 18 (36.0 \%) & \\
\hline Secundigravida & 47 (31.3\%) & 17 (34.0\%) & 10 (20.0\%) & 20 (40.0\%) & \\
\hline multigravida & $59(39.3 \%)$ & 21 (42.0 \%) & 26 (52.0\%) & 12 (24.0\%) & \\
\hline \multicolumn{6}{|l|}{ Family history of HTN } \\
\hline Yes & $22(14.7 \%)$ & 1 (2.0\%) & $4(8.0 \%)$ & 17 (34.0\%) & $<0.0001$ \\
\hline \multicolumn{6}{|l|}{ History of abortion } \\
\hline Yes(spontaneous) & 75 (50.0 \%) & $21(42.0 \%)$ & $22(44.0 \%)$ & $32(64.0 \%)$ & 0.0288 \\
\hline \multicolumn{6}{|c|}{ Previous caesarean section } \\
\hline Yes & 39 (26.0 \%) & $6(12.0 \%)$ & $9(18.0 \%)$ & $24(48.0 \%)$ & $<0.0001$ \\
\hline \multicolumn{6}{|l|}{$\mathrm{BP}(\mathrm{mmHg})$} \\
\hline SBP & $146.1 \pm 1.5$ & $114.3 \pm 1.0$ & $158.8 \pm 1.9^{b}$ & $165.2 \pm 1.6^{b}$ & 0.0020 \\
\hline DBP & $93.8 \pm 1.1$ & $69.33 \pm 1.0$ & $103.4 \pm 1.2^{b}$ & $108.6 \pm 1.1^{\mathrm{b}}$ & 0.0079 \\
\hline Urinary protein (g/l) & $0.7 \pm 0.1$ & $0.01 \pm 0.0$ & $0.150 \pm 0.0^{\mathrm{a}}$ & $2.05 \pm 0.1^{a}$ & $<0.0001$ \\
\hline
\end{tabular}

Values are presented as frequency (proportion) and mean \pm SD. $p<0.05$ is considered statistically significant difference. ${ }^{a}$ significant compared to NP; ${ }^{b}$ significant compared to NP

\section{Baseline and postpartum levels of angiogenic regulators} and oxidative stress biomarkers

Median levels of sFlt-1, 8-epi-PGF2 $\alpha$ and sFlt-1/ PLGF were significantly elevated in $\mathrm{PE}>\mathrm{GH}>\mathrm{NP}$ $(p<0.0001)$ whilst levels of PLGF, T-AOC and PLGF/ sFlt-1 were significantly reduced in $\mathrm{PE}>\mathrm{GH}>\mathrm{NP}$ $(p<0.0001)$ before delivery. Median levels of PLGF, PLGF/sFlt-1 ratio and T-AOC increased in PE, GH and NP at $48 \mathrm{~h}$ after delivery compared levels before delivery $(p<0.0001)$ whilst levels of sFlt-1, sFlt-1/ PLGF ratio and 8-epi-PGF2 $\alpha$ decreased at $48 \mathrm{~h}$ after delivery in $\mathrm{PE}, \mathrm{GH}$, and NP compared to levels before delivery $(p<0.0001)$ (Table 2$)$.

\section{Antepartum and postparturm obstetric outcomes}

Table 3 shows antepartum and postpartum obstetric complications and outcomes among study participants. A greater proportion of study participants had cephalic presentation. A significant higher proportion of preeclamptics $(24.0 \%)$ had breech presentation compared to NP $(0.0 \%)$ and GH (8.0\%). There were increased proportion of placental praevia $(14.0 \%$ vs $0.0 \%)$, placental abruptio (22.0\% vs $0.0 \%$ ), IUFD (40.0\% vs $0.0 \%$ ), IUGR (44.0\% vs $1.0 \%)$, APH (22.0\% vs $0.0 \%)$ and PPROM (16.0\% vs $0.0 \%)$ in PE compared to NP control $(p<0.05)$. Significantly, higher proportion $(86.0 \%)$ of PE and $28.0 \%$ of $\mathrm{GH}$ were delivered by emergency caesarean section 
Table 2 Baseline and Postpartum levels of Angiogenic and oxidative biomarkers among the studied groups

\begin{tabular}{|c|c|c|c|c|c|c|}
\hline \multirow[t]{2}{*}{ Parameters } & \multicolumn{2}{|l|}{ NP } & \multicolumn{2}{|l|}{$\mathrm{GH}$} & \multicolumn{2}{|l|}{$\mathrm{PE}$} \\
\hline & Pre-delivery & $\begin{array}{l}48 \mathrm{~h} \\
\text { postpartum }\end{array}$ & Pre-delivery & $\begin{array}{l}48 \mathrm{~h} \\
\text { postpartum }\end{array}$ & Pre-delivery & $\begin{array}{l}48 \mathrm{~h} \\
\text { postpartum }\end{array}$ \\
\hline \multicolumn{7}{|l|}{ Angiogenic Factors } \\
\hline \multirow[t]{2}{*}{ PLGF (pg/ml) } & 137.7 & $187.9^{* *}$ & 53.05 & $163.9^{* * *}$ & 21.40 & $96.3^{* * *}$ \\
\hline & (88.1-177.8) & $(130.7-214.6)$ & $(38.33-74.80)$ & (94.00-200.3) & $(12.40-48.40)$ & $(77.3-131.0)$ \\
\hline \multirow[t]{2}{*}{ sFlt-1 (pg/ml) } & 119.5 & $45.6^{* * *}$ & 413.2 & $77.3^{* * *}$ & 787.6 & $104.6^{* * *}$ \\
\hline & (87.4-160.4) & $(19.28-88.7)$ & $(164.5-635.0)$ & $(44.4-101.6)$ & $(510.7-926.3)$ & $(66.3-139.0)$ \\
\hline \multirow[t]{2}{*}{ sFlt-1/PLGF } & 0.9 & $0.2^{* * *}$ & 7.8 & $0.5^{* * *}$ & 36.80 & $1.1^{* * *}$ \\
\hline & $(0.6-1.2)$ & $(0.2-0.5)$ & $(5.4-12.8)$ & $(0.2-0.7)$ & $(16.42-56.92)$ & $(0.3-2.0)$ \\
\hline \multirow[t]{2}{*}{ PLGF/sFlt-1 } & 1.1 & $3.4^{* *}$ & 0.1 & $2.2^{* * *}$ & 0.0351 & $0.9^{* * *}$ \\
\hline & $(0.5-2.0)$ & $(1.9-9.5)$ & $(0.1-0.5)$ & $(1.0-3.7)$ & $(0.015-0.085)$ & $(0.5-1.7)$ \\
\hline \multicolumn{7}{|l|}{ Oxidative biomarkers } \\
\hline \multirow[t]{2}{*}{ 8-epi-PGF2a (pg/ml) } & 35.7 & $15.8^{* *}$ & 198.8 & $18.4^{* * *}$ & 324.6 & $66.3^{* * *}$ \\
\hline & $(28.7-42.9)$ & $(12.2-18.7)$ & $(92.48-392.3)$ & $(12.0-32.1)$ & $(194.2-618.6)$ & $(32.65-100.4)$ \\
\hline \multirow[t]{2}{*}{ TAOC (mmol/l) } & 1.1 & $1.2^{*}$ & 0.6 & $0.8^{*}$ & 0.5 & $0.7^{* * *}$ \\
\hline & $(1.0-1.2)$ & $(1.1-1.3)$ & $(0.4-0.8)$ & $(0.6-1.1)$ & $(0.2-0.6)$ & $(0.4-0.9)$ \\
\hline
\end{tabular}

Values are presented as median (Interquartile range). ${ }^{*} p<0.05 ;{ }^{* *} p<0.001 ;{ }^{* * *} p<0.0001$

compared to NP controls $(0.0 \%)$. Presentations such as fresh stillbirth (24.0\% vs $2.0 \%)$, PPH (26.0\% vs $6.0 \%)$, preterm delivery (90.0 \% vs $6.0 \%$ ) and maternal mortality (10.0\% vs $0.0 \%$ ) were significantly associated with PE pregnancies compared to NP controls. However, prolonged obstructed labour was mostly common in $\mathrm{GH}$ pregnancies compared NP pregnancies (58\% vs $10.0 \%$ ).

\section{Angiogenic and oxidative stress levels categorized to severity of the condition}

Figure 1 summarizes the levels of angiogenic and oxidative stress markers in relation to severity of pregnancy condition. Median levels of PLGF, PLGF/sFlt-1 and TAOC decreased in the order of severity (severe $\mathrm{PE}<\mathrm{PE}<$ $\mathrm{GH}<\mathrm{NP} ; p<0.0001)$ whilst sFlt-1, 8-epi-PGF2 $\alpha$ and sFlt$1 /$ PLGF ratio significantly increased in study participants in the order of severity (severe PE $>\mathrm{PE}>\mathrm{GH}>\mathrm{NP} ; p<0.0001$ ) (Fig. 1).

\section{Angiogenic and oxidative stress levels in relation to adverse pregnancy outcomes in PE}

Figure 2 shows the levels of angiogenic and oxidative stress markers in PE and PE co-associated adverse outcome. Median Levels of sFlt-1, 8-epi-PGF2 $\alpha$ and sFlt-1/ PLGF were significantly elevated among study participants with PE co-existing with IUFD, placental abruptio, placental previa, HELLP syndrome and IUGR compared to $\mathrm{PE}$ without adverse outcome $(p=0.041, p=0.005$, $p=0.0002)$. Levels of PLGF, T-AOC and PLGF/sFlt-1 were significantly reduced in study participants with $\mathrm{PE}$ co-existing with IUFD, placental abruptio, placental previa, HELLP syndrome and IUGR compared to PE without adverse outcome $(p=0.0013, p=0.006, p<0.0001$ respectively) (Fig. 2).

\section{Birthweight of babies according to studied groups}

As shown in Fig. 3, babies of pregnant women with PE associated IUGR had significantly low birthweight (LBW) compared to those with only PE, GH, and NP $(p<0.0001)$ (Fig. 3).

Correlation of angiogenic and oxidative biomarkers with antepartum and postpartum adverse pregnancy outcome in PE

Table 4 shows Spearman rho correlation of angiogenic and oxidative stress biomarkers with antepartum and adverse maternal outcomes in preeclamptic pregnancy. There was a significant $(p<0.05)$ negative correlation of PLGF and T-AOC with IUGR, placental abruptio, IUFD, stillbirth, and $\mathrm{PPH}$. Conversely, there was a significant positive correlation $(p<0.05)$ of sFlt- 1 and 8-epi-PGF $2 \alpha$ with IUGR, placental abruptio, IUFD, stillbirth, and PPH (Table 4).

\section{Correlation of angiogenic and oxidative biomarkers with antepartum and postpartum adverse pregnancy outcome in $\mathrm{GH}$}

Table 5 shows Spearman rho correlation of angiogenic and oxidative stress biomarkers with antepartum and adverse maternal outcomes in GH. In general there were no significant correlation between angiogenic 
Table 3 0-Antepartum and Postpartum obstetric characteristic and adverse complications

\begin{tabular}{|c|c|c|c|}
\hline Variables & $\mathrm{NP}(n=50)$ & $\mathrm{GH}(n=50)$ & $\mathrm{PE}(n=50)$ \\
\hline \multicolumn{4}{|l|}{ Fetal presentation } \\
\hline CephalicNetex & $50(100.0 \%)$ & 46(92.0 \%) & $38(76.0 \%)$ \\
\hline breech & $0(0.0 \%)$ & $4(8.0 \%)$ & $12(24.0 \%)$ \\
\hline \multicolumn{4}{|l|}{ Placental praevia } \\
\hline Yes & $0(0.0 \%)$ & $4(8.0 \%)$ & $7(14.0 \%)$ \\
\hline \multicolumn{4}{|l|}{ Placental abruption } \\
\hline Yes & $0(0.0 \%)$ & $1(2.0 \%)$ & $11(22.0 \%)$ \\
\hline \multicolumn{4}{|l|}{ IUFD } \\
\hline Yes & $0(0.0 \%)$ & $5(10.0 \%)$ & $20(40.0 \%)$ \\
\hline \multicolumn{4}{|l|}{ IUGR } \\
\hline Yes & $1(2.0 \%)$ & $7(14.0 \%)$ & $22(44.0 \%)$ \\
\hline \multicolumn{4}{|l|}{$\mathrm{APH}$} \\
\hline Yes & $0(0.0 \%)$ & $2(8.0 \%)$ & $11(22.0 \%)$ \\
\hline \multicolumn{4}{|l|}{ PPROM } \\
\hline Yes & $0(0.0 \%)$ & $0(0.0 \%)$ & $8(16.0 \%)$ \\
\hline \multicolumn{4}{|l|}{ Mode of delivery } \\
\hline \multicolumn{4}{|l|}{ Vaginal } \\
\hline Spontaneous & 45(90.0 \%) & 15(30.0 \%) & $0(0.0 \%)$ \\
\hline Induced & $5(10.0 \%)$ & $20(40.0 \%)$ & $1(2.0 \%)$ \\
\hline \multicolumn{4}{|l|}{ Caesarean section } \\
\hline Emergency & $0(0.0 \%)$ & $14(28.0 \%)$ & $43(86.0 \%)$ \\
\hline Elective & $0(0.0 \%)$ & $1(2.0 \%)$ & 7(14.0\%) \\
\hline \multicolumn{4}{|l|}{ Stillbirth } \\
\hline Fresh & $1(2.0 \%)$ & $5(10.0 \%)$ & $12(24.0 \%)$ \\
\hline Macerated & $0(0.0 \%)$ & $1(2.0 \%)$ & $2(4.0 \%)$ \\
\hline \multicolumn{4}{|l|}{ Live birth } \\
\hline Yes & 49(98.0 \%) & 44(88.0 \%) & $36(72.0 \%)$ \\
\hline \multicolumn{4}{|l|}{ Prolong labour } \\
\hline Yes & $5(10.0 \%)$ & $29(58.0 \%)$ & $13(26.0 \%)$ \\
\hline \multicolumn{4}{|l|}{ PPH } \\
\hline Yes & $3(6.0 \%)$ & $5(10.0 \%)$ & 13(26.0\%) \\
\hline \multicolumn{4}{|l|}{ Status of delivery } \\
\hline Term & 47(94.0 \%) & $12(24.0 \%)$ & $5(10.0 \%)$ \\
\hline preterm & $3(6.0 \%)$ & $38(76.0 \%)$ & $45(90.0 \%)$ \\
\hline \multicolumn{4}{|l|}{ Maternal mortality } \\
\hline Yes & $0(0.0 \%)$ & $0(0.0 \%)$ & $5(10.0 \%)$ \\
\hline
\end{tabular}

Values are presented as frequency (proportion) unless otherwise indicated

factor, oxidative stress biomarkers and adverse pregnancy outcomes among GH subjects $(p>0.05)$.

\section{Discussion}

Hypertension in pregnancy especially preeclampsia has been linked with maternal mortality, premature birth, IUGR, stillbirth and other adverse outcomes [3, 7].
However, the mechanism linking adverse pregnancy outcomes and preeclampsia is unknown. This study assessed the correlation between angiogenic regulators and oxidation stress markers with adverse pregnancy outcomes among Ghanaian preeclamptic and gestational hypertensive women. Imbalance in angiogenic regulators was identified by an increased in sFlt-1 with a corresponding decreased in PLGF levels among PE subjects with complications. Oxidative stress was observed amongst the participants with GH, PE and PE co-existing with adverse pregnancy outcomes as depicted by the high lipid peroxidation and reduced TAOC levels. Furthermore, angiogenic and oxidative stress biomarkers correlated significantly with IUGR, IUFD, placental abruptio, stillbirth and PPH. The findings of this study has demonstrated that women with preeclampsia complicated by IUGR had a markedly widespread endothelial dysfunction depicted by the extremely elevated sFlt-1 and reduced PLGF levels compared to normal pregnancy and PE without associated complications (Fig. 2). A previous study by Ghosh et al. [19] observed reduced levels of PLGF in PE but not others [11]. Imbalance in angiogenic regulators leading to placental bed hypoxia and a subsequent endothelial dysfunction may have ultimately resulted in IUGR [19]. Our finding shows that preeclamptic pregnancy complicated by IUGR is one of the probable causes of low birth weight (LBW) infants (Fig. 3). Again the findings of this study shows a significantly elevated sFlt-1 and a corresponding decreased PLGF levels observed in PE women with IUFD, placental previa, placental abruptio and HELLP syndrome compared to those with PE only have not been reported by previous studies. The mechanism of these finding though not well understood, an overall reduction of foetal perfusion of the placenta and shallow trophoblasts invasion have been implicated [19]. The present study indicates that angiogenic growth factors may be reliable biomarkers for the prediction of adverse pregnancy outcomes (IUGR, IUFD, placental abruptio, stillbirth, and PPH). Biochemical imbalance in preeclampsia may be associated with an increase oxidative stress depicted by increased lipid peroxidation as well as a defective antioxidant protection. In addition to the defective angiogenic regulators, elevated 8-epiPGF2 $\alpha$ and reduced TAOC were significantly linked with IUGR, IUFD, placental previa, placental abruptio and HELLP syndrome pregnancies in this present study. PE associated with adverse outcomes may have created disequilibria in the levels of pro-oxidants (increased 8-epiPGF $2 \alpha$ ) and antioxidants proportionate to the degree of severity (reduced TAOC) culminating in oxidative stress. Significantly high lipid peroxidation and a compromised antioxidant system emphasizes that oxidative stress play a pivotal role in the etiology of adverse pregnancy outcome. 

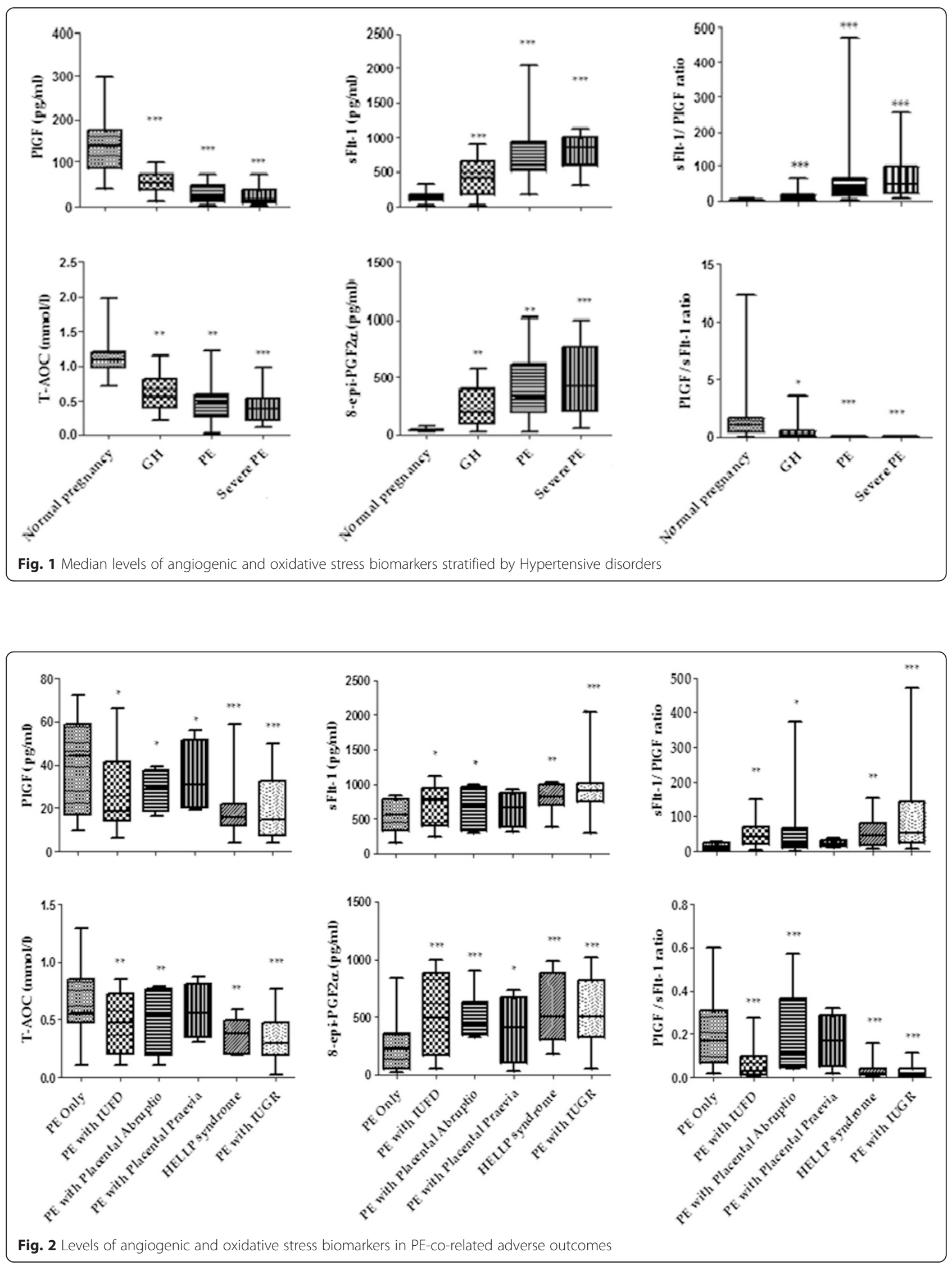


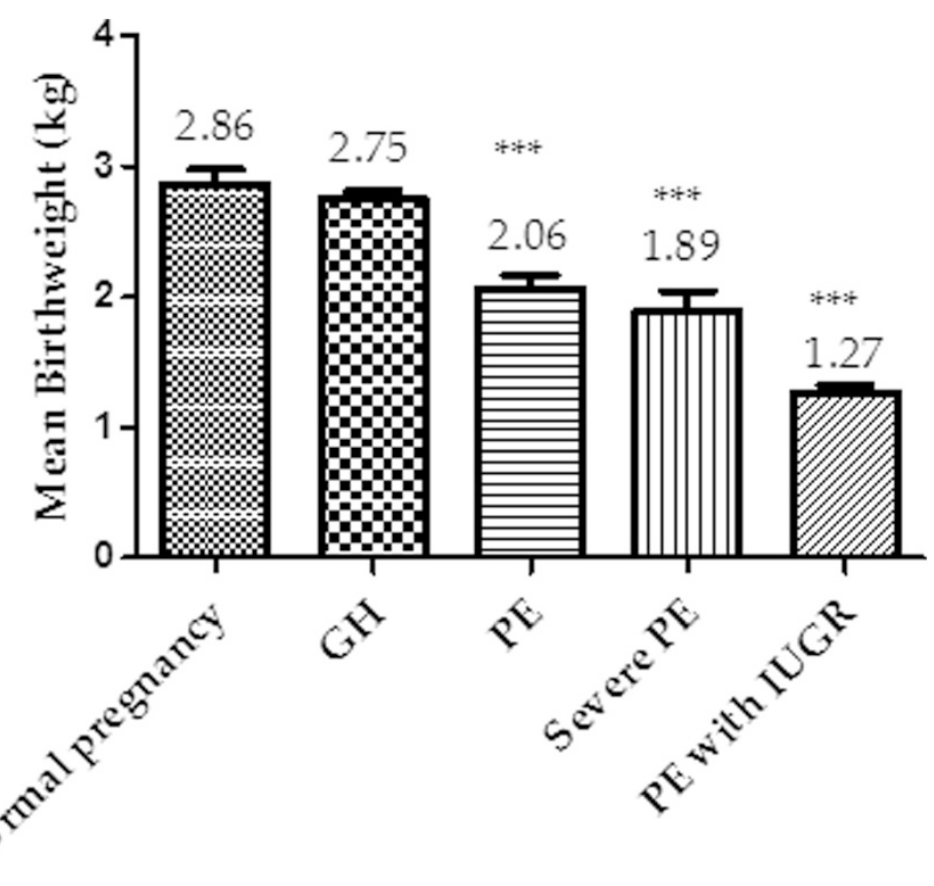

Fig. 3 Birthweight of babies stratified by the severity of the condition

Some studies $[11,19]$ indicated that proangiogenic and anti-angiogenic proteins may be useful biomarkers in predicting pregnancy complications such as preeclampsia and fetal growth restriction. Ghosh et al. [19] have reported a significant negative correlation between PIGF and IUGR which is consistent with the present study (Table 4). This study is the first to observe a significant negative correlation between PLGF and IUFD, placental abruptio, stillbirth, and PPH and a positive correlation between sFlt-1 and IUGR, IUFD, placental abruptio, stillbirth, and PPH. This probably indicates that the degree of sFlt-1 is elevated while PLGF is reduced proportionately to the hypertensive pregnancy complicated with adverse outcomes. The markedly increased sFlt-1 and corresponding decreased PLGF levels proportional to the severity of PE condition indicates that imbalances in angiogenic regulators play an important role in the etiology of these adverse outcomes. Hypoxia induced by incomplete trophoblasts invasion and placental underperfusion may explain these findings [19].

Table 4 Spearman rho correlation of Angiogenic and Oxidative biomarkers with antepartum and postpartum adverse pregnancy outcome in Preeclampsia women

\begin{tabular}{|c|c|c|c|c|}
\hline & PLGF & sFlt-1 & 8-epi-PGF2a & T-AOC \\
\hline \multicolumn{5}{|l|}{ Antepartum complications } \\
\hline \multirow[t]{2}{*}{ IUGR } & $r=-0.416$ & $r=0.549$ & $r=0.610$ & $r=-0.635$ \\
\hline & $p=0.0030$ & $p<0.0001$ & $p<0.0001$ & $p<0.0001$ \\
\hline \multirow[t]{2}{*}{ Placental abruptio/praevia } & $r=-0.663$ & $r=0.680$ & $r=0.579$ & $r=-0.740$; \\
\hline & $p<0.0001$ & $p<0.0001$ & $p<0.0001$ & $p<0.0001$ \\
\hline \multirow[t]{2}{*}{ IUFD } & $r=-0.694$ & $r=0.730$ & $r=0.629$ & $r=-0.819$ \\
\hline & $p<0.0001$ & $p<0.0001$ & $p<0.0001$ & $p<0.0001$ \\
\hline \multicolumn{5}{|l|}{ Postpartum adverse outcomes } \\
\hline \multirow[t]{2}{*}{ Stillbirth } & $r=-0.366$ & $r=0.732$ & $r=0.691$ & $r=-0.815$ \\
\hline & $p=0.0183$ & $p<0.0001$ & $p<0.0001$ & $p<0.0001$ \\
\hline \multirow[t]{2}{*}{$\mathrm{PPH}$} & $r=-0.297$ & $r=0.479$ & $r=0.410$ & $r=-0.509$ \\
\hline & $p=0.0420$ & $p=0.0043$ & $p=0.0039$ & $p=0.0044$ \\
\hline
\end{tabular}


Table 5 Spearman rho correlation of Angiogenic and Oxidative biomarkers with antepartum and postpartum adverse pregnancy outcome in Gestational hypertensive women

\begin{tabular}{|c|c|c|c|c|}
\hline & PLGF & sFlt-1 & 8-epi-PGF2a & T-AOC \\
\hline \multicolumn{5}{|l|}{ Antepartum complications } \\
\hline \multirow[t]{2}{*}{ IUGR } & $r=-0.109$ & $r=0.027$ & $r=0.009$ & $r=-0.203$ \\
\hline & $p=0.060$ & $p=0.901$ & $p=0.725$ & $p=0.197$ \\
\hline \multirow[t]{2}{*}{ Placental abruptio/praevia } & $r=-0.033$ & $r=0.040$ & $r=0.022$ & $r=-0.107$ \\
\hline & $p=0.683$ & $p=0.259$ & $p=0.901$ & $p=0.443$ \\
\hline \multirow[t]{2}{*}{ IUFD } & $r=-0.191$ & $r=0.150$ & $r=0.083$ & $r=-0.196$ \\
\hline & $p=0.071$ & $p=0.092$ & $p=0.724$ & $p=0.173$ \\
\hline \multicolumn{5}{|l|}{ Postpartum adverse outcomes } \\
\hline \multirow[t]{2}{*}{ Stillbirth } & $r=-0.066$ & $r=0.001$ & $r=0.113$ & $r=-0.191$ \\
\hline & $p=0.483$ & $p=0.933$ & $p=0.270$ & $p=0.420$ \\
\hline \multirow[t]{2}{*}{$\mathrm{PPH}$} & $r=-0.057$ & $r=0.019$ & $r=0.181$ & $r=-0.159$ \\
\hline & $p=0.183$ & $p=0.396$ & $p=0.317$ & $p=0.158$ \\
\hline
\end{tabular}

r: correlation coefficient; $p<0.05$ (statistically significant) $p<0.001$ (statistically highly significant) $p<0.0001$ (statistically very highly significant). $r<0.5$ (weak correlation); $r>0.5$ (strong correlation)

The present study has established a relationship between oxidative stress as indicated by high 8 -epiPGF $2 \alpha$ and low TAOC with adverse pregnancy outcome (Table 4). As 8 -epiPGF2 $\alpha$ correlated positively with adverse pregnancy outcome (IUGR, IUFD, placental abruptio, stillbirth, and $\mathrm{PPH}$ ) in preeclamptic participants, the correlation of TAOC with IUGR, IUFD, placental abruptio, stillbirth, and PPH levels was significant and inversely correlated. However, no significant correlation between adverse pregnancy outcome and markers of angiogenic factors and oxidative stress biomarkers were observed among women presenting with $\mathrm{GH}$ in this study (Table 5).

PE associated adverse outcome may be using up the antioxidant proteins in circulation for its metabolic processes and this could explain the reduced concentration of TAOC. Adverse pregnancy outcome were mostly associated with preeclamptic pregnancies than gestational hypertension indicating that pregnancies complicated by high blood pressure and proteinuria stands an increased risk of suffering these adverse outcomes than normal pregnancy and GH. The increase in sFlt-1 and 8-epiPGF $2 \alpha$ in PE is an indication that these markers may play a synergistic role in the pathogenesis of $\mathrm{PE}$ and may be responsible for the widespread endothelial dysfunction culminating in these adverse pregnancy outcomes. The limitation of this study was the small sample size used. However, the imbalance in the levels of angiogenic factors and oxidative stress biomarkers corresponded well with some previous studies. This study will serve as a baseline and thus further studies with prospective cohort design involving large sample size will be needed to establish the strength of this observation.

\section{Conclusion}

$\mathrm{PE}$ is associated with an imbalance in the angiogenic regulators (increased sFlt-1 and decreased PLGF) and oxidative markers (increased 8-epiPGF2 $\alpha$ and decreased TAOC) proportionate to the adverse pregnancy outcomes. Early identification of these imbalance would alert health care givers in anticipation of a possible adverse pregnancy outcome and thus increased surveillance during pregnancy and parturition and put in the necessary preventive measures to ameliorate the adverse outcome.

\section{Abbreviations}

GH: Gestational hypertension; PE: Preeclampsia; PLGF: Placental growth factor (PIGF); sFlt-1: Soluble fms-like tyrosine kinase 1; 8-epi-PGF2a: 8-epiprostaglandin F2alpha; T-AOC: Total antioxidant capacity; ROS: Reactive oxygen species; IUGR: Intrauterine growth restriction; IUFD: Intrauterine fetal death; PPH: Postpartum haemorrhage; PPROM: Premature prerupture of membranes; NP: Normal pregnancy.

\section{Competing interests}

The authors of this manuscript declare that there is no competing interest with regards to this article.

\section{Authors' contributions}

Conceived and designed the experiments: EOA, WKBAO, and SAS. Performed the experiments: EOA WKBAO, SAS and TCA Analyzed the data: EOA, RKDE Wrote the first draft of the manuscript: EOA, SAS, and WKBAO Contributed to the writing of the manuscript: EOA, SAS, WKBAO and RKDE. Agree with manuscript results and conclusions: EOA, SAS, WKBAO and RKDE. Enrolled patients: EOA and TCA. All authors read and approved the final manuscript.

\section{Acknowledgement}

The authors would like to acknowledge the Department of O\&G, KATH and Department of Molecular medicine for endorsing this study. We also wish to thank the Midwife for their immense support during sample collection. We also would like to thank Medilab diagnostic centre, Kumasi for they help in biochemical analysis. We wish to thank the study participants. 


\section{Author details}

'Department of Molecular Medicine, School of Medical Sciences, Kwame Nkrumah University of Science and Technology (KNUST), Kumasi, Ghana. ${ }^{2}$ Department of Obstretics \& Gynaecology, Komfo Anokye Teaching Hospital, Kumasi, Ghana. ${ }^{3}$ Department of Bacteriology, Noguchi Memorial Institute for Medical Research, Accra, Ghana. ${ }^{4}$ Department of Medical Laboratory

Technology, University of Cape Coast, Cape Coast, Ghana.

Received: 20 February 2015 Accepted: 17 August 2015

Published online: 25 August 2015

\section{References}

1. Walker JJ. Pre-eclampsia. Lancet. 2000;356(9237):1260-5.

2. Suplee PD, Dawley K, Bloch JR. Tailoring peripartum nursing care for women of advanced maternal age. J Obstet Gynecol Neonatal Nurs. 2007;36(6):616-23.

3. Huang L, Sauve R, Birkett N, Fergusson D, van Walraven C. Maternal age and risk of stillbirth: a systematic review. Can Med Assoc J. 2008;178(2):165-72.

4. Palm M. Oxidative Stress, Angiogenesis and Inflammation in Normal Pregnancy and Postpartum. Acta Universitatis Upsaliensis Digital comprehensive summaries of Uppasala Dissertations from the Faculty of Medicine Uppsala ISBN 978-91-554-8314-2. 2012:753-763.

5. Petla LT, Chikkala R, Ratnakar KS, Kodati V, Sritharan V. Biomarkers for the management of pre-eclampsia in pregnant women. Indian J Med Res. 2013;138(1):60.

6. Smith GCS, Crossley JA, Aitken DA, Jenkins N, Lyall F, Cameron AD, et al. Circulating angiogenic factors in early pregnancy and the risk of preeclampsia, intrauterine growth restriction, spontaneous preterm birth, and stillbirth. Obstet Gynecol. 2007;109(6):1316-24.

7. Elkholi DGEY, Nagy HM. Maternal serum concentrations of angiogenic and antiangiogenic factors in threatened miscarriage at 7-12 weeks' gestation and the risk of adverse pregnancy outcomes. Middle East Fertility Society Journal. 2013;18(3):208-13.

8. Chen S-F, Lo L-M, Li M-J, Yeh Y-L, Hung T-H. The association between maternal oxidative stress at mid-gestation and subsequent pregnancy complications. Reprod Sci. 2012;19(5):505-12.

9. ACOG Committee on Practice Bulletins-Obstetrics. ACOG practice bulletin. Diagnosis and management of preeclampsia and eclampsia. Obstet Gynecol. 2002;99:159.

10. Kirkendall WM, Burton AC, Epstein FH, Freis ED. Recommendations for human blood pressure determination by sphygmomanometers. Circulation. 1967;36(6):980-8.

11. Schlembach D, Wallner W, Sengenberger R, Stiegler E, Mörtl M, Beckmann MW et al. Angiogenic growth factor levels in maternal and fetal blood: correlation with Doppler ultrasound parameters in pregnancies complicated by preeclampsia and intrauterine growth restriction. Ultrasound Obstet Gynecol. 2007;29(4):407-13.

12. Ohkuchi A, Hirashima C, Matsubara S, Suzuki H, Takahashi K, Arai F, et al. Alterations in placental growth factor levels before and after the onset of preeclampsia are more pronounced in women with early onset severe preeclampsia. Hypertens Res. 2007;30(2):151-9.

13. Lawn JE, Gravett MG, Nunes TM, Rubens CE, Stanton C. Global report on preterm birth and stillbirth (1 of 7): definitions, description of the burden and opportunities to improve data. BMC pregnancy and childbirth. 2010;10 Suppl 1:S1.

14. World Health Organization. International statistical classification of diseases and related health problems, vol. 1: World Health Organization; 2004.

15. Sh J, Ghiyasi $P$, Haghani $H$. Risk factors related to intra uterine fetal death in Iran, A case-control study. Shiraz E-Medical journal. 2005;6:3-4.

16. Oyelese Y, Ananth CV. Placental abruption. Obstet Gynecol. 2006;108(4):1005-16.

17. Oyelese Y, Smulian JC. Placenta previa, placenta accreta, and vasa previa Obstet Gynecol. 2006;107(4):927-41.

18. Caughey AB, Robinson JN, Norwitz ER. Contemporary diagnosis and management of preterm premature rupture of membranes. Rev Obstet Gynecol. 2008;1(1):11.

19. Ghosh SK, Raheja S, Tuli A, Raghunandan C, Agarwal S. Low Serum placental growth Factor Levels in late second trimester can act as biomarker for predicting intra uterine growth retardation in pregnancies complicated by preeclampsia Baixos niveis de plgF no segundo trimestre da gravidez. Acta obstet ginecol port. 2012;6(5):51-7.

\section{Submit your next manuscript to BioMed Central and take full advantage of:}

- Convenient online submission

- Thorough peer review

- No space constraints or color figure charges

- Immediate publication on acceptance

- Inclusion in PubMed, CAS, Scopus and Google Scholar

- Research which is freely available for redistribution

Submit your manuscript at www.biomedcentral.com/submit 